Chrischta Ganz

\title{
QuinMedica-Kongress 2017: Thema «Das Geschlecht macht den Unterschied/ Gendermedizin und Ernährung»
}

Nach dem Erfolg in den Jahren 2013 und 2015 fand am 23. und 24. September 2017 in Winterthur zum dritten Mal der QuinMedica-Kongress für Naturheilkunde, Alternativund Komplementärmedizin statt. Mit dem Ziel, den Wissensaustausch in der Naturheilkunde und ganzheitlichen Medizin zu fördern, hatten die beiden Organisatoren Heide-Dore und Thomas Bertschi-Stahl Ärzte/Ärztinnen, Apotheker/Apothekerinnen, Naturheilpraktiker/Naturheilpraktikerinnen und Therapeuten/Therapeutinnen zum zweitägigen Kongress eingeladen.

Zum Thema «Das Geschlecht macht den Unterschied/Gendermedizin und Ernährung» boten Referierende aus dem deutschsprachigen Raum den Kongressteilnehmern grosses Fachwissen und neueste Erkenntnisse. Die Kongressthemen waren gut gewählt, ist doch die Frage der Gendermedizin (endlich) Diskussionspunkt in medizinischen Fachkreisen. Auch die Ernährung mit ihren unendlich vielen, zum Teil völlig verschiedenen Empfehlungen ist ein umstrittenes, hart umkämpftes Thema, gerade auch in Fachkreisen.

\section{Das Geschlecht macht den Unterschied/Gendermedizin}

Unter Gendermedizin (Gender Medicine), auch geschlechtsspezifische Medizin/Gendermedizin genannt, versteht man eine Humanmedizin, die (neben den sozialen und psychologischen) den biologischen Unterschieden von Frau und Mann besondere Aufmerksamkeit schenkt und eine geschlechtsabhängige Erforschung von Krankheiten und deren Behandlung fordert. Aus ganzheitlicher Sicht ist die differenzierte Betrachtung von Mann und Frau ein Muss, da Ganzheitsmedizin den Menschen immer individuell betrachtet. Etwas salopp formuliert, ist es jedem therapeutisch arbeitenden Menschen vollkommen klar, dass eine feingliedrige, $50 \mathrm{~kg}$ schwere Frau nicht die gleiche Behandlung benötigt wie ein muskulöser, handwerklich tätiger Mann mit 90 kg Körpergewicht (auch wenn auf der Packungsbeilage die Dosierung für jeden Erwachsenen z.B. mit 3 $\times 1$ Tablette angegeben wird). Heute ist erwiesen, dass Frauen sehr viel stärker unter Nebenwirkungen von Arzneimitteln leiden als Männer, was nicht alleine auf den Unterschied des Körpergewichtes zurückzuführen ist.

In den 1990er-Jahren entwickelte sich die Gendermedizin als Teilgebiet einer personalisierten Medizin. Marianne Legato, eine amerikanische Kardiologin, Medizinwissenschaftlerin und Autorin («Evas Rippe», Fachzeitschrift Gender Medicine), wies schon 1980 auf die Unterschiede von Herzerkrankungen bei Frauen und Männern hin. Erst 2001 empfahl die Weltgesundheitsorganisation (WHO), eine geschlechtsspezifische Medizin $\mathrm{zu}$ entwickeln.

Neben dem gesellschaftlichen Interesse an der Genderfrage (man erinnere sich an «Warum Männer nicht zu- hören und Frauen schlecht einparken»), das in den letzten zwei Jahrzehnten stark zugenommen hat, gibt es auch gut erforschte, medizinische Erkenntnisse - beispielsweise, dass bei Frauen stärkere Entzündungsreaktionen (aufgrund stärkerer Immunantwort) und damit verbunden eine höhere Anzahl Autoimmunerkrankungen oder aber bei Männern eine höhere Suchttendenz beobachtet werden. Am bekanntesten ist heute aber mit Sicherheit der Unterschied der Herzinfarkt-Symptome bei Frauen und Männern.

Frauen und Männer unterscheiden sich - diese Unterschiede sind ein beinahe unerschöpfliches Thema, das häufig sehr kontrovers diskutiert wird. Dr. Damir del Monte, Lehrbeauftragter für Funktionelle Neuroanatomie an den Universitäten Heidelberg und Innsbruck, zeigte in seinem humorvollen und gleichzeitig ernsthaften Referat ( Von sexy Gehirnen und der Kunst des Einparkens») Unterschiede auf und räumte gleichzeitig mit einigen falschen Aussagen auf: So ist beispielsweise das Gerücht, dass Frauen schlechter einparken, nie wissenschaftlich bestätigt worden. Das Dilemma jedoch bleibt: Einerseits bestätigen diverse Studien, dass die Geschlechterrolle das Ergebnis sozialer Konstrukte und das biologische Geschlecht relativ unbedeutend ist. Andererseits finden sich in den physischen Gegebenheiten eines männlichen oder weiblichen Körpers immens viele Unterschiede, die eine Gendermedizin geradezu aufdrängen. Es

\section{KARGER}

(c) 2017 S. Karger GmbH, Freiburg

Fax +497614520714 


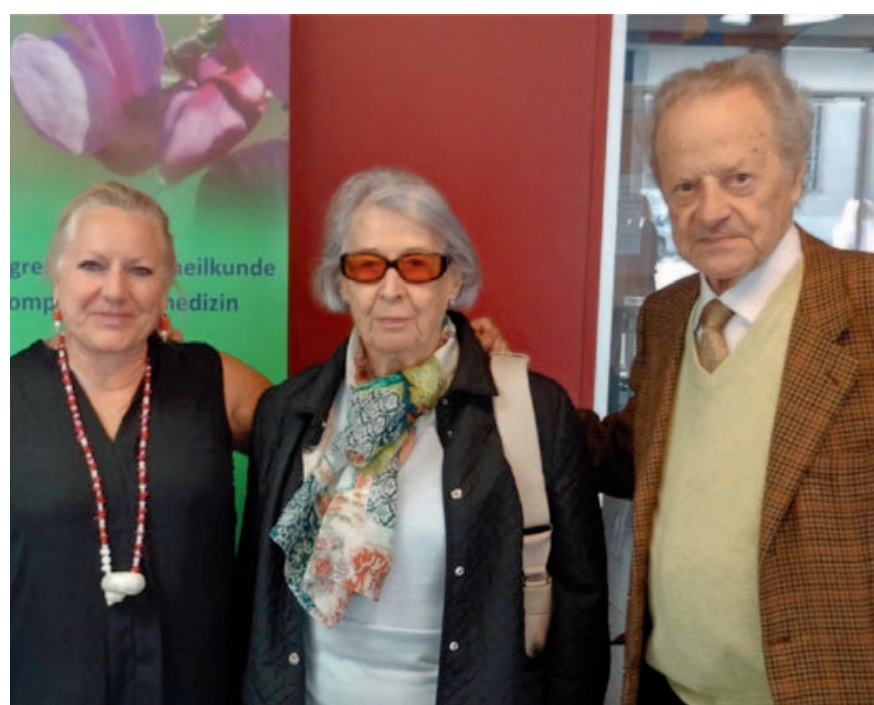

Abb. 1. Heide-Dore Bertschi-Stahl (links) konnte auch Natale Ferronato (rechts) und seine Frau Maryse Ferronato (Mitte) für den Kongress gewinnen (Foto: $\mathrm{zVg}$ )

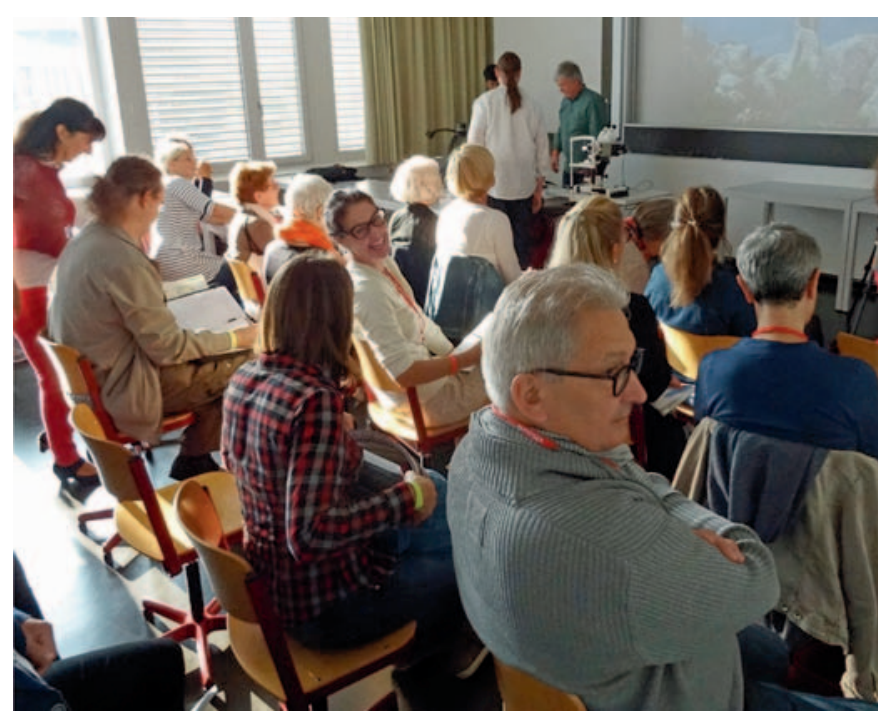

Abb. 2. Interessierte Fachpersonen am Vortrag «Irisdiagnose nach der Humoralmedizin» von Friedemann Garvelmann (Foto: zVg). lohnt sich darum für alle medizinischen Fachpersonen, in der täglichen Praxis bewusste Therapiestrategien aufgrund geschlechtsspezifischer Unterschiede zu wählen.

Dr. med. Simon Feldhaus, Allgemeinmediziner sowie stellvertretender Chefarzt und Leiter medizinische Dienste Paramed-Ambulatorium Baar, hingegen schwächte in seinem Referat «Gender Medizin - Macht alleine das Geschlecht den Unterschied?» die Unterscheidung Mann/Frau ab und betonte, dass jeder Mensch verschieden ist und infolgedessen auch jeder Mensch individuell behandelt werden muss.

Trotzdem zeigte Feldhaus unbestrittene Unterschiede von Mann und Frau auf, die Einfluss auf Gesundheit, Krankheit und Heilung haben: beispielsweise Muskelmasse, Fettanteil und Fettverteilung, Körpergrösse, Leberstoffwechsel und der weibliche Zyklus, der mit seinen monatlichen Schwankungen den Gesamtstoffwechsel der Frau völlig anders gestaltet als beim Mann.

Abschliessend betonte Feldhaus, dass Medizin immer persönlich abgestimmt sein muss und nicht stereotyp ausgewählt werden darf, und rückte darum die Individualmedizin ohne ein Festhalten an «So ist der Mann und so ist die Frau» in den Vordergrund.

\section{Ernährung}

Neben dem Thema «Gendermedizin» stand die Ernährung im Fokus. Sybille Binder, dipl. Ernährungsberaterin HF und dipl. Vitalstofftherapeutin, zeigte in ihrem Referat «Hormone und Ernährung bei Mann und Frau», wie Hormone den Körper steuern. Ebenso beleuchtete sie den umgekehrten Weg: Körperliche Aspekte regulieren ihrerseits die Hormone. Aus naturheilkundlicher Sicht kann darum niemals nur eine einzelne Hormondrüse bzw. ihre Dysfunktion die Therapie bestimmen, denn alle Hormondrüsen kommunizieren miteinander (was nichts Neues ist, aber in der Medizin immer wieder vernachlässigt wird). Binder zeigte in ihrem Vortrag die Verbindung von Hormonsystem, Nervensystem und Emotionen auf. Beispielsweise ist ein erhöhter Cholesterinspiegel ein (mögliches) Zeichen dafür, dass der Körper mehr Cholesterin zur Synthese von Stress- und Sexualhormonen benötigt. Die alleinige medikamentöse Senkung eines erhöhten Cholesterinspiegels kann darum nie eine ganzheitliche Therapie sein. Vielmehr geht es darum zu erkennen, was die Ursache für einen erhöhten Cholesterinspiegel ist.

Für einen optimal funktionierenden Hormonhaushalt muss der Mensch über die Nahrung zwingend genügend hochwertige Fettsäuren, Eiweisse und Vitalstoffe aufnehmen. Aus Sicht der Traditionellen Europäischen Naturheilkunde (TEN) steht dabei eine gute Assimilation im Verdauungstrakt im Vordergrund - in der TEN Kochung (Coctio) genannt. Die Kochung ist der bestimmende Faktor, ob der Mensch die körperfremde Nahrung in körpereigene Substanzen zu verwandeln vermag. Ähnlich wie man es aus der Traditionellen Chinesischen Medizin oder aus dem Ayurveda kennt, ist in der TEN eine gut funktionierende Verdauung das $\mathrm{A}$ und $\mathrm{O}$ für die Gesundheit bzw. für die Genesung im Krankheitsfall. Eine angepasste Ernährung und die Unterstützung einer guten Verdauung sind darum in jeder Therapie die Basis des Behandlungskonzeptes. Dies gilt auch für hormonelle Fehlfunktionen. Eine alleinige Supplementierung von Vitalstoffen, Fettsäuren usw. reicht darum in der Praxis oft nicht aus. 
Ein weiterer wichtiger Aspekt ist, dass der Mensch, wenn es ihm nicht gut geht (Stress, Unwohlsein, Ärger), vermehrt auf Zucker, Alkohol sowie anregende Nahrungsmittel wie Koffein zurückgreift, was den Hormonhaushalt mittel- und langfristig massiv stört. Sexualdrüsen, Pankreas, Nebennieren, Schilddrüse und alle anderen Hormondrüsen werden dadurch in ihrer endokrinen Funktion geschwächt, im schlimmsten Fall bis zu einer Dysfunktion.

\section{Weitere Referate}

Weitere Referenten waren Dr. med. Petra Wiechel («Wenn das Herz bricht»), Volker Ohland ( $\mathrm{JCH}$ - die intelligente Ölmischung für eine zukunftsfähige Medizin»), Natale Ferronato («Wie wird man im Dschungel der Ernährung bald 92 Jahre alt?») (Abb. 1), Hermann Bichsel («Immunmodulation nach Dr. Spengler - Therapievorschläge»), Jesse de Groodt («Revolutionäre spektralanalytische Analyse»), Friedemann Garvelmann («Unsere Familienplanung ist abgeschlossen - was jetzt?» sowie «Irisdiagnose nach der Humoralmedizin») (Abb. 2), Jürgen Malchers («Fit und gesund bis ins hohe Alter»), Franz Amann («Elektrosmog - was meinen meine Zellen dazu?»), Andrea Chiappa («Fasten in den Wechseljahren»), Max Geiser («Allergien effizient mit Bioresonanz behandeln»), Manfred J. Poggel ( Parkinson-Männer - Parkinson-Frauen - Wege zur optimalen Ernährung»), Rolf Ott («Wenn die Wirbelsäule nicht im Lot ist»), Dr. med. Teelke Beck ( Sinn und Unsinn von Nahrungsergänzungsmitteln»), Roland Zehntner («Energiemangel - die Ursache jeder Erkrankung»), Ursula Ehrhorn («Analyse des vegetativen
Nervensystems»), Prof. Dr. Friedrich Hainbuch ( GGendermedizin: Kräuterund Bienenprodukte»), Jascha Hutter («Mikrozirkulation - die physikalische Gefässtherapie»), Eva Candrian («Wahl der passenden Partner-Apotheke»), Andreas Bachmair («HPVImpfung auch für die Knaben?»), Dr. med. Boris Müller-Hübenthal («Integrative Onkologie oder die Quadratur des Kreises»), Heidi Schönenberger («Naturheilpraktiker/in mit eidg. Diplom: Was bedeutet das?»), Dr. med. Susanne Römer («Weibliche Psychosomatik - immer wieder überraschend»), Arnold Mayr («Das weibliche Prinzip - physiologische und pathologische Aspekte»), Renate Poggel («Wege zur optimalen Ernährung») und Dr. Renato Kaiser («Revision Heilmittelgesetz - was gilt für die Alternativ- und Komplementärmedizin?»).

\section{Podiumsdiskussion}

Die Podiumsdiskussion zum Thema «Wie wichtig ist der kleine Unterschied?» mit Andreas Chiappa, Dr. med. Boris Müller-Hübenthal, Dr. iur. Michael Nonn, Dr. med. Petra Wiechel und Heide-Dore Bertschi-Stahl (Moderation: Andréas Härry (Abb. 3)) rundete den ersten Kongresstag ab. Ob nun die Unterscheidung Mann/ Frau oder eher die Individualität des einzelnen Menschen im Fokus steht, beurteilten die Diskussionsteilnehmer unterschiedlich. Zurück zum «Vergleich» der feingliedrigen, $50 \mathrm{~kg}$ schweren Frau mit dem muskulösen, handwerklich tätigen Mann mit einem Körpergewicht von $90 \mathrm{~kg}$ am Anfang dieses Beitrags: Ob die Unterschiede nun geschlechterspezifisch oder aber auf individuelle Konstitution zurückzuführen sind, lässt sich nicht eindeu-

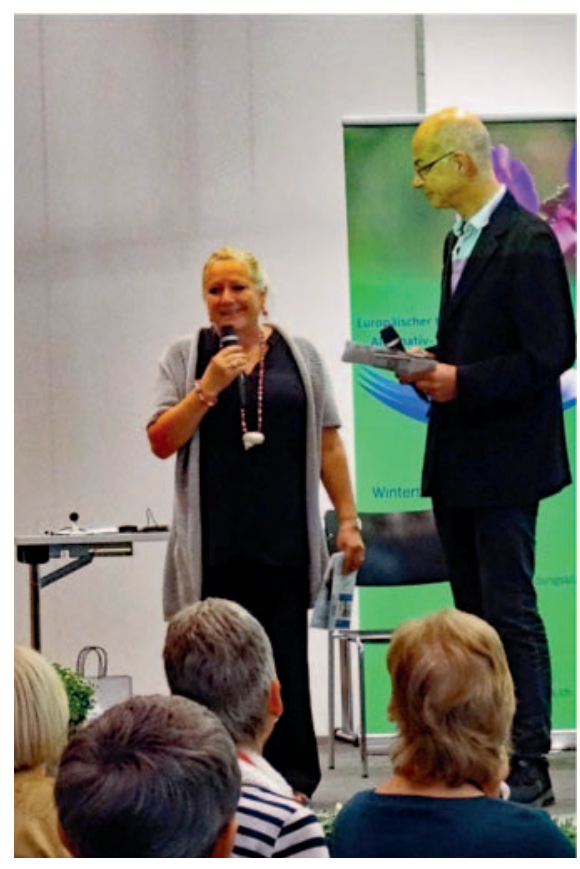

Abb. 3. Heide-Dore Bertschi-Stahl und der Moderator Andréas Härry (Foto: $\mathrm{zVg}$ ).

tig klären - diese Frage muss wohl von jeder medizinischen Fachperson persönlich beantwortet werden.

Dr. med. Petra Wiechel schloss die Diskussion mit den Worten: «Wir haben genug geredet, nun gilt es zu handeln!»

\section{Ausblick}

Die äusserst freundliche und gleichzeitig professionelle Atmosphäre während der beiden Kongresstage bot einen guten Rahmen für eine bereichernde fachliche Weiterbildung, persönliche Gespräche und die Vernetzung von Fachleuten aus den verschiedensten Disziplinen. Darum lohnt es sich auch, das Datum des nächsten QuinMedica-Kongresses diesmal zum Thema «Der suchende Patient» - am 5. und 6. April 2019 in Luzern zu reservieren. 